\title{
Elevated prothrombin time/international normalized ratio associated with concurrent administration of regorafenib and warfarin in a patient with advanced colorectal cancer
}

\author{
Hironori Kitade ${ }^{1 *}$, Azusa Hiromasa-Yamasaki ${ }^{1}$, Kengo Hokkoku' ${ }^{1}$, Mitsue Mori ${ }^{1}$, Michio Watanabe ${ }^{2,3}$, \\ Masuo Nakai ${ }^{2}$ and Seiji Yano 3,4
}

\begin{abstract}
Background: Regorafenib and its metabolites may inhibit the activities of several CYP or UDP-glucuronosyltransferase isoforms, including that of CYP2C9. Therefore, pharmacological agents that are CYP2C9 substrates may show elevated circulating levels and enhanced drug efficacy when concurrently used with regorafenib. Previous studies showed that the area under the plasma concentration-time curve of warfarin, which is the substrate for CYP2C9, increased upon co-administration of regorafenib. However, there are no reports indicating that the anticoagulant effects of warfarin increased upon co-administration of regorafenib.

Case presentation: We report a case of a 76-year-old man with liver metastasis of colon cancer. He was treated with regorafenib at a dosage of $120 \mathrm{mg}$ daily on days 1 to 21 every 4 weeks as a third-line therapy. He had a history of acute myocardial infarction and had taken $2 \mathrm{mg}$ warfarin daily. Three weeks after the treatment began, PT/INR values markedly increased, although there was no hemorrhage. Administration of regorafenib and warfarin was discontinued, and then PT/INR rapidly decreased. Warfarin administration was restarted ( $0.5 \mathrm{mg}$ daily) and the dose was increased up to $1.5 \mathrm{mg}$ daily. The patient's PT/INR values exhibited a tendency to increase when concurrently used with regorafenib, the dose of which was reduced to $80 \mathrm{mg}$ daily on days 1 to 14 every 3 weeks at a physician's discretion.

Conclusions: The clinical course of this patient suggested that PT/INR might increase during concurrent use of warfarin and regorafenib. Therefore, PT/INR should be periodically monitored during the concurrent use of warfarin and regorafenib.
\end{abstract}

Keywords: Regorafenib, Warfarin, PT/INR, Colorectal cancer

\section{Background}

Regorafenib is an oral multikinase inhibitor, which has shown antitumor activity in patients with advanced colorectal cancer or gastrointestinal stromal tumors who have previously received standard treatment and discontinued it owing to disease progression or side effects $[1,2]$. Regorafenib is mainly metabolized to $M-2$ and $M-5$ by

\footnotetext{
* Correspondence: hiro.kitacchi@gmail.com

1Department of Pharmacy, Houju Memorial Hospital, 11-71, Midorigaoka,

Nomi-shi, Ishikawa 923-1226, Japan

Full list of author information is available at the end of the article
}

cytochrome P-450 (CYP) 3A4, both of which have pharmacological activity similar to that of regorafenib [3]. In addition, results from in vitro analysis showed that regorafenib and its metabolites might inhibit the activity of several CYPs such as CYP2C9 and CYP2C8 or UDPglucuronosyltransferase isoforms [3].

The plasma concentration of warfarin, the substrate for CYP2C9, could be increased with the concurrent use of regorafenib, which leads to enhance warfarin's anticoagulant effects. Pharmacokinetic studies have shown that the area under the plasma concentration-time curve 
of warfarin increases with concurrent administration of regorafenib [3]. However, there are no reports indicating that regorafenib interacts with warfarin and leads to increased anticoagulant effects. Here, we report the case of a patient with advanced colon cancer, who experienced elevated prothrombin time/international normalized ratio (PT/INR) with concurrent use of regorafenib and warfarin.

\section{Case presentation}

A 76-year-old man with stage IV colon cancer metastasis underwent resection of part of the colon and cholecystectomy. He had a history of hypothyroidism and acute myocardial infarction. He did not drink alcohol but had a history of smoking a pack of cigarettes daily for about 40 years. He had taken medicines, including levothyroxine (75 $\mu \mathrm{g}$ daily), spironolactone ( $25 \mathrm{mg}$ daily), nicorandil (10 mg daily), pravastatin (5 mg daily), allopurinol (200 mg daily), and warfarin ( $2 \mathrm{mg}$ daily). After surgery, treatment was started with modified FOLFOX-6 (mFOLFOX6, $85 \mathrm{mg} / \mathrm{m}^{2}$ oxaliplatin, $200 \mathrm{mg} / \mathrm{m}^{2}$ leucovorin, $400 \mathrm{mg} / \mathrm{m}^{2} 5$-FU bolus on day 1 and $2400 \mathrm{mg} / \mathrm{m}^{2} 5-\mathrm{FU}$ over 46 h every 2 weeks) in July 2012 to liver metastasis. His PT/INR value was controlled around 2.0 before receiving mFOLFOX6 and did not increase when this treatment was given. He was received mFOLFOX6 for 6 months, but his carcinoembryonic antigen (CEA) level increased, and computed tomography (CT) showed progression of liver metastasis. Then, treatment with irinotecan (CPT-11) plus S-1 was initiated (CPT-11 $125 \mathrm{mg} / \mathrm{m}^{2}$ on days 1 and 15 and S-1 $40 \mathrm{mg}$ twice daily for 2 weeks, repeated every 4 weeks). His PT/INR value slightly increased to 2.98 from 2.312 weeks after CPT-11 plus S-1 was administered. Then the dose of warfarin was reduced to $1 \mathrm{mg}$ and PT/INR value decreased to around 2.0. However, after 3 cycles of treatment with CPT- 11 plus S-1, he developed interstitial pneumonia and discontinued this therapy, leading to decreased PT/INR value around 1.3. Furthermore, the interstitial pneumonia improved but a CT scan indicated that his hepatic metastasis had progressed. Then, he began a course of treatment with regorafenib at a dosage of $120 \mathrm{mg}$ daily on days 1 to 21 every 4 weeks in October 2013. The warfarin dose was increased up to $1.5 \mathrm{mg}$ one month before initiation of treatment with regorafenib and up to $2 \mathrm{mg}$ again when regorafenib was given concurrently (Fig. 1).

Although the patient's PT/INR value was 1.26 before initiation of treatment with regorafenib, this value significantly increased to 2.1 on day $8,2.98$ on day 15 , and 6.4 on day 22 (Fig. 1). Regorafenib and warfarin treatments were discontinued, and his PT/INR value decreased to 1.31 within 1 week after the discontinuation. Then, warfarin was reduced by $0.5 \mathrm{mg}$ daily and was restarted. As he had developed grade 2 hand-foot syndrome during the first cycle of treatment, regorafenib was also reduced by $80 \mathrm{mg}$ daily on days 1 to 14 every 3 weeks at a physician's discretion and was restarted.

The warfarin dose was increased up to $1 \mathrm{mg}$ daily after 2 weeks and up to $1.5 \mathrm{mg}$ daily after 2 months, respectively. Although his PT/INR value increased gradually with an increasing dose of warfarin, further elevation was not seen with the concomitant use of regorafenib. Additionally, laboratory findings showed that liver functions including alanine transaminase and aspartate transaminase serum levels were normal, although the liver metastasis showed slight progression on a CT scan (data not shown).

In March 2014, regorafenib and warfarin treatment were discontinued again because the patient developed a pneumothorax. One month later, the pneumothorax healed and the patient was restarted on a course of warfarin (1.5 mg daily) prior to regorafenib. Two weeks later, regorafenib treatment was also restarted at the same treatment schedule and dose, at which time his $\mathrm{PT} / \mathrm{INR}$ value was 2.04 . His $\mathrm{PT} / \mathrm{INR}$ value increased to 3.37 again on day 8 after restarting treatment with regorafenib (Fig. 1). He was then discharged with the treatment of $1 \mathrm{mg}$ warfarin daily. The PT/INR value reduced to 2.37 one week after the reduction of warfarin treatment. Then, the PT/INR value did not increase during the concomitant use of regorafenib. However, his CEA level increased, and his hepatic metastasis showed progression on a CT scan. Then, treatment with regorafenib was discontinued and the patient was treated with trifluridine and tipiracil hydrochloride (Lonsurf $f^{\circ}$ ). PT/INR value did not increase when the salvage treatment with Lonsurf ${ }^{\oplus}$ was given (data not shown).

This case report was approved by the ethics review committee of the Houju Memorial Hospital (Ishikawa, Japan) (No. 16-5).

\section{Discussion}

This is the first report of a patient with advanced colon cancer who had a significant increase in the PT/INR when administered warfarin concomitantly with regorafenib. Although the warfarin dose was increased up to $2 \mathrm{mg}$ when regorafenib was administered simultaneously, the patient did not demonstrate a marked increase in PT/INR with a daily $2 \mathrm{mg}$ dose of warfarin before receiving chemotherapy. Therefore, we suggest that this event was not caused only by an increase in the dose of warfarin.

There is a possibility that impaired hepatic function due to tumor progression results in the elevation of PT/ INR, but no deterioration of liver function was observed, while hepatic metastasis had slightly progressed during the regorafenib treatment. In addition, it is suggested that regorafenib itself may increase the PT/INR value directly via inhibition of vascular endothelial growth 


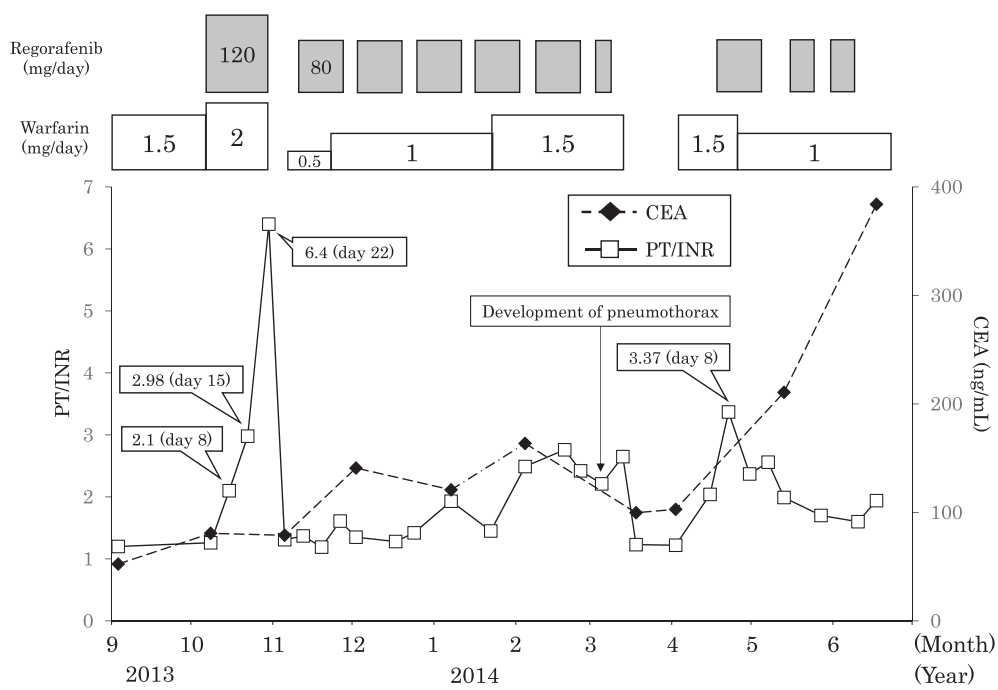

Fig. 1 Prothrombin time/international normalized ratio (PT/INR) values and serum carcinoembryonic antigen (CEA) levels during regorafenib treatment. Only an important points are mentioned PT/INR values (day on course) after co-administration of regorafenib

factor signaling, which is related to the induction of angiogenesis. Indeed, a previous report showed that INR value increased in 120 out of 500 patients (24 \%) who were treated with regorafenib [3]. However, in this case, the PT/INR value did not increase when a low dose of warfarin (0.5-1 mg daily) was administered, whereas its value increased at the warfarin dose was increased up to 1.5-2 mg, while the dose of regorafenib was same (Fig. 1). Therefore, we speculate that PT/INR increase in this case was not caused by direct action of regorafenib but by drug interaction between regorafenib and warfarin.

We assessed this event using the Naranjo adverse drug reaction probability scale, which indicated a probable relationship (score of 6) between regorafenib use and the elevated PT/INR value [4]. The Naranjo scale was designed to evaluate single drug adverse events, not drug interactions. We also assessed the causation of this adverse reaction using the drug interaction probability scale score for potential drug interactions in the patient, which indicated a probable likelihood of an interaction (score of 7) [5].

Warfarin is highly bound (99\%) to human plasma proteins [6]. In vitro protein binding of regorafenib, M2 , and M-5 to human plasma proteins is also high at 99.5, 99.8, and $99.95 \%$, respectively [3]. There were no change in serum albumin level during concomitant use of regorafenib and warfarin in our case (data not shown). Although we could not measure the plasma free levels of warfarin or regorafenib and its metabolite, it is possible that the concentration of plasma-free warfarin will be elevated and lead to increased anticoagulant effects via competition for protein binding sites when these two drugs are used concurrently. However, it is unknown that which metabolite is most contribute to the displacement warfarin from plasma proteins.

Warfarin is metabolized via several CYPs, including CYP3A4, with involvement of CYP1A1, CYP1A2, CYP2C8, CYP2C9, CYP2C18 and CYP2C19 and CYP3A4 [7-10]. S-warfarin, which exhibits potent anticoagulant activity, is metabolized primarily by CYP2C9 [7]. Regorafenib and its metabolite $\mathrm{M}-2$ inhibit CYP2C9 [3]. $\mathrm{IC}_{50}$ values for CYP2C9 inhibition by regorafenib and M-2 are 2.7 and $6.1 \mu \mathrm{M}$, respectively [11]. Furthermore, pharmacokinetic analysis from a phase I study also revealed that after oral administration of $160 \mathrm{mg}$ or $120 \mathrm{mg}$ regorafenib for 3 weeks, the plasma $C_{\max }$ value in its steady state of regorafenib is slightly higher than that of M-2 [12, 13]. In this case, the patient was not administered regorafenib concomitantly with a potent inducer or inhibitor of CYP3A4, which is the main enzyme involved in metabolism of regorafenib. Therefore, although we could not measure plasma $\mathrm{C}_{\max }$ values of regorafenib and $\mathrm{M}-2$ at the steady state, we speculate that the steady state $C_{\max }$ value of regorafenib in this case could also be larger than that of M-2. Therefore, we speculate that regorafenib may have more potent inhibitory effect on CYP2C9 than M-2, considering $\mathrm{IC}_{50}$ and steady state $\mathrm{C}_{\max }$ values of them.

In this case, a significant elevation of PT/INR occurred 3 weeks after regorafenib co-administration. Goto et al. reported that hematuria and a marked increase in PT/ INR occurred after administering warfarin for about 1 month [14]. In this report, PT/INR increased due to CYP2C9 variants because the patient had the CYP2C9*3/*3 allele. Indeed, CYP2C9 genotypes are closely associated with warfarin dose adjustment [15]. Of the more than 30 identified CYP2C9 variants, the CYP2C9*3 allele is associated with reduced enzyme 
activity and requires lower maintenance doses of warfarin [16]. Obayashi et al. reported that the mean daily dose of warfarin was lower in subjects with CYP2C9:1/ *3 (1.86 $\pm 0.80 \mathrm{mg}$ daily) than in subjects with CYP2C9*1/*1 (3.36 $\pm 1.43 \mathrm{mg}$ daily) in a Japanese population [17]. A similar result was reported by Ohno et al. for differences in the mean maintenance dose of warfarin in Japanese patients with CYP2C9 variants [18]. Although we did not analyze the CYP2C9 variant in our case, the PT/INR value was controlled with $2 \mathrm{mg}$ warfarin daily before receiving chemotherapy. Thus, we speculate that the patient might have the CYP2C9*3 allele, which might contribute to an increase in the PT/ INR after the concomitant administration of regorafenib.

Moreover, it has been reported that other oral multikinase inhibitors, such as imatinib, sorafenib, and vemurafenib, when used concurrently with warfarin, may also have the ability to increase circulating levels of warfarin and potentiating its anticoagulant effects [19-21]. Therefore, when treatment with such oral multikinase inhibitors is administered to patients who receive warfarin daily, switching from warfarin to other novel oral anticoagulants such as rivaroxaban and apixaban based on drug interaction should be considered.

\section{Conclusions}

The clinical course of this patient suggested that the PT/ INR increases during the concurrent use of warfarin and regorafenib. Therefore, PT/INR should be periodically monitored during the concurrent use of warfarin and regorafenib.

\section{Abbreviations}

CEA, carcinoembryonic antigen; CPT-11, irinotecan; CT, computed tomography; CYP, cytochrome P-450; mFOLFOX6, modified FOLFOX-6; PT/INR, prothrombin time/international normalized ratio

\section{Acknowledgements}

The authors thank all staff members of Houju Memorial Hospital that were provided the patient's data in this case report.

\section{Funding}

There are no funding sources for this case report.

\section{Availability of data and materials}

Data used in this case report will not be shared due to the risk of identifying an individual, although most patient's data are presented in the main paper.

\section{Authors' contributions}

HK wrote the manuscript. AH-Y, KH, MM, MW, MN, and SY helped to draft the manuscript. All authors read and approved the final manuscript.

\section{Competing interests}

The authors declare that they have no competing interests.

\section{Consent for publication}

Written informed consent was obtained from the patient's next of kin for publication of this case report and any accompanying images. A copy of the written consent is available for review by the Editor-in-Chief of this journal.

\section{Author details}

'Department of Pharmacy, Houju Memorial Hospital, 11-71, Midorigaoka, Nomi-shi, Ishikawa 923-1226, Japan. ${ }^{2}$ Department of Gastroenterology, Houju Memorial Hospital, 11-71, Midorigaoka, Nomi-shi, Ishikawa 923-1226, Japan. ${ }^{3}$ Outpatient Cancer Chemotherapy Center, Houju Memorial Hospital, 11-71, Midorigaoka, Nomi-shi, Ishikawa 923-1226, Japan. ${ }^{4}$ Department of Medical Oncology, Cancer Research Institute, Kanazawa University, 13-1 Takara-machi, Kanazawa, Ishikawa 920-0934, Japan.

Received: 10 April 2016 Accepted: 20 June 2016

Published online: 08 July 2016

\section{References}

1. Grothey A, Cutsem EV, Sobrero A, Siena S, Falcone A, Ychou M, et al. Regorafenib monotherapy for previously treated metastatic colorectal cancer (CORRECT): an international, multicentre, randomised, placebocontrolled, phase 3 trial. Lancet. 2013;381:303-12.

2. Demetri GD, Reichardt P, Kang YK, Blay JY, Rutkowski P, Gelderblom H, et al. Efficacy and safety of regorafenib for advanced gastrointestinal stromal tumours after failure of imatinib and sunitinib (GRID): an international, multicentre, randomised, placebo-controlled, phase 3 trial. Lancet. 2013;381:295-302.

3. U.S. Food and Drug Administration: Stivarga ${ }^{\oplus}$ (regorafenib). 2015. http:// www.accessdata.fda.gov/drugsatfda_docs/label/2015/203085s004lbl.pdf. Accessed 12 Jan 2016.

4. Naranjo CA, Busto U, Sellers EM, Sandor P, Ruiz I, Roberts EA, et al. A method for estimating the probability of adverse drug reactions. Clin Pharmacol Ther. 1981;30:239-45.

5. Horn JR, Hansten PD, Chan LN. Proposal for a new tool to evaluate drug interaction cases. Ann Pharmacother. 2007;41:674-80.

6. U.S. Food and Drug Administration: Coumadin (warfarin sodium). 2011. http://www.accessdata.fda.gov/drugsatfda_docs/label/2011/009218s107lbl. pdf. Accessed 10 Mar 2016.

7. Rettie AE, Korzekwa KR, Kunze KL, Lawrence RF, Eddy AC, Aoyama T, et al. Hydroxylation of warfarin by human CDNA-expressed cytochrome P-450: a role for P-4502C9 in the etiology of (S)-warfarin-drug interactions. Chem Res Toxicol. 1992;5:54-9.

8. Ngui JS, Chen Q, Shou M, Wang RW, Stearns RA, Baillie TA, et al. In vitro stimulation of warfarin metabolism by quinidine: increases in the formation of 4'- and 10-hydroxywarfarin. Drug Metab Dispos. 2001;29:877-86.

9. Zhang Z, Fasco MJ, Huang Z, Guengerich FP, Kaminsky LS. Human cytochromes P4501A1 and P4501A2: R-warfarin metabolism as a probe. Drug Metab Dispos. 1995;23:1339-46.

10. Wienkers $L C$, Wurden CJ, Storch E, Kunze KL, Rettie AE, Trager WF. Formation of (R)-8-hydroxywarfarin in human liver microsomes. A new metabolic marker for the (S)-mephenytoinhydroxylase, P4502C19. Drug Metab Dispos. 1996;24:610-4

11. Australian public assessment report for Regorafenib. 2014. https://www.tga. gov.au/sites/default/files/auspar-regorafenib-140207.pdf. Accessed 26 Apr 2016.

12. Mross K, Frost A, Steinbild S, Hedbom S, Büchert M, Fasol U, et al. A phase I dose-escalation study of regorafenib (BAY 73-4506), an inhibitor of oncogenic, angiogenic, and stromal kinases, in patients with advanced solid tumors. Clin Cancer Res. 2012;18:2658-67.

13. Strumberg D, Scheulen ME, Schultheis B, Richly H, Frost A, Buchert M, et al. Regorafenib (BAY 73-4506) in advanced colorectal cancer: a phase I study. Br J Cancer. 2012;106:1722-7.

14. Goto T, Miura M, Murata A, Terata K, Uno T, Yamamoto K, et al. Standard warfarin dose in a patient with the CYP2C9*3/*3 genotype leads to hematuria. Clin Chim Acta. 2010;411:1375-7.

15. Higashi MK, Veenstra DL, Kondo LM, Wittkowsky AK, Srinouanprachanh SL, Farin FM, et al. Association between CYP2C9 genetic variants and anticoagulation-related outcomes during warfarin therapy. JAMA. 2002;287: $1690-8$.

16. Niinuma Y, Saito T, Takahashi M, Tsukada C, Ito M, Hirasawa N, et al. Functional characterization of 32 CYP2C9 allelic variants. Pharmacogenomics J. 2014;14:107-14.

17. Obayashi K, Nakamura K, Kawana J, Ogata H, Hanada K, Kurabayashi M, et al. VKORC1 gene variations are the major contributors of variation in warfarin dose in Japanese patients. Clin Pharmacol Ther. 2006;80:169-78. 
18. Ohno M, Yamamoto A, Ono A, Miura G, Funamoto M, Takemoto Y, et al. Influence of clinical and genetic factors on warfarin dose requirements among Japanese patients. Eur J Clin Pharmacol. 2009;65:1097-103.

19. U.S. Food and Drug Administration: Gleevec (imatinib mesylate). 2012. http:// www.accessdata.fda.gov/drugsatfda_docs/label/2012/021588s035lbl.pdf. Accessed 15 Mar 2016.

20. Moretti LV, Montalvo RO. Elevated International Normalized Ratio associated with concurrent use of sorafenib and warfarin. Am J Health Syst Pharm. 2009;66:2123-5

21. U.S. Food and Drug Administration: Zelboraf ${ }^{\oplus}$ (vemurafenib). 2015. http:// www.accessdata.fda.gov/drugsatfda_docs/label/2015/202429s008lbl.pdf. Accessed 15 Mar 2016

Submit your next manuscript to BioMed Central and we will help you at every step:

- We accept pre-submission inquiries

- Our selector tool helps you to find the most relevant journal

- We provide round the clock customer support

- Convenient online submission

- Thorough peer review

- Inclusion in PubMed and all major indexing services

- Maximum visibility for your research

Submit your manuscript at www.biomedcentral.com/submit
Biomed Central 